\title{
Mesenteric Panniculitis in Sjögren's Syndrome: A New Systemic Manifestation to Consider?
}

\author{
Patrícia Afonso Mendes ${ }^{1,2}$, Diana Marques Ferreira ${ }^{1,2}$, Helena Temido $^{1,2}$, Rui Pina $^{1,2}$, Armando de Carvalho $^{1,2}$ \\ ${ }^{1}$ Department of Internal Medicine A, Coimbra Hospital and University Center, Coimbra, Portugal \\ ${ }^{2}$ University Clinic of Internal Medicine, Faculty of Medicine, University of Coimbra, Coimbra, Portugal
}

\section{Doi: 10.12890/2018_000788- European Journal of Case Reports in Internal Medicine - ○ EFIM 2018}

Received: $16 / 11 / 2017$

Accepted: $29 / 01 / 2018$

Published: $14 / 02 / 2018$

How to cite this article: Mendes P, Ferreira D, Temido H, Pina R, de Carvalho A. Mesenteric panniculitis in Sjögren's syndrome: a new systemic manifestation to consider? EJCRIM 2018;5: doi:10.12890/2018_000788.

Conflicts of Interests: The Authors declare that there are no competing interests.

This article is licensed under a Commons Attribution Non-Commercial 4.0 License

\section{ABSTRACT}

The association between mesenteric panniculitis and Sjögren's syndrome, although rare, is starting to be recognized. Usually, mesenteric panniculitis is symptomatic, presenting with either general or gastrointestinal symptoms. Sjögren's syndrome is an autoimmune disease that typically affects secretory glands, but may have serious systemic involvement. We report the case of a 77-year-old patient in whom accidental discovery of asymptomatic mesenteric panniculitis on computed tomography led to the diagnosis of Sjögren's syndrome with several systemic manifestations.

\section{LEARNING POINTS}

- Mesenteric panniculitis is a rare condition, sometimes associated with autoimmune diseases, which can make the differential diagnosis with more frequent systemic diseases difficult. There is no specific treatment for mesenteric panniculitis, but it is agreed that only symptomatic patients should be treated.

- Although Sjögren's syndrome typically presents with xerostomia and xerophthalmia (dry eyes and mouth syndrome, DEMS), it should always be kept in mind that systemic manifestations can occur, especially in more severe cases.

\section{KEYWORDS}

Sjögren's syndrome, mesenteric panniculitis, autoimmune diseases

\section{BACKGROUND}

The prevalence of mesenteric panniculitis (MP) is unknown due to under-diagnosis. MP can present as an acute or chronic fibrotic and inflammatory process that affects the mesentery. Its aetiology is unknown but can be related to surgery or trauma, autoimmune diseases, neoplasms, infection or ischaemic injury. This condition can present as abdominal pain, obstruction, weight loss or, in most cases, may be asymptomatic ${ }^{[1]}$. Primary Sjögren's syndrome (SS) is an autoimmune disease mainly known for lymphocytic infiltration of the secretory glands (xerostomia and xerophthalmia, also called DEMS (dry eyes and mouth syndrome), is the common presentation), but it can occur with systemic manifestations like interstitial lung disease, Raynaud's phenomenon and arthritis $^{[2]}$ or, in rare cases, mesenteric panniculitis ${ }^{[3-6]}$. 


\section{CASE DESCRIPTION}

A 77-year-old man was referred to the internal medicine clinic for investigation to determine the aetiology of asymptomatic mesenteric panniculitis (Fig. 1A,B). A computed tomography (CT) scan performed because of raised liver enzymes revealed MP. The patient reported he had experienced asymmetrical tricolour Raynaud's phenomenon since adolescence, with skin thickening over the digits but no digital ulcers. He also complained of xerophthalmia without xerostomia, but had no other symptoms or complaints (including abdominal pain or other symptoms of the digestive system).

The patient had a personal history of type 2 diabetes mellitus, hypertension, hyperuricemia, mixed dyslipidaemia, overweight, chronic alcoholism (approximately $96 \mathrm{~g}$ alcohol/day) and previous cholecystectomy, and was a former smoker (he had stopped 25 years previously). On physical examination, the patient exhibited malar telangiectasia and parotid hypertrophy, skin thickening of the distal phalanxes of the fingers, nail clubbing and asymmetrical bicolour Raynaud's phenomenon (Fig. 2). Pulmonary auscultation revealed bilateral dry crackles. The differential diagnosis included neoplastic diseases and mesenteric panniculitis due to previous surgery, but also autoimmune diseases such as systemic sclerosis, SS and mixed connective tissue disease.

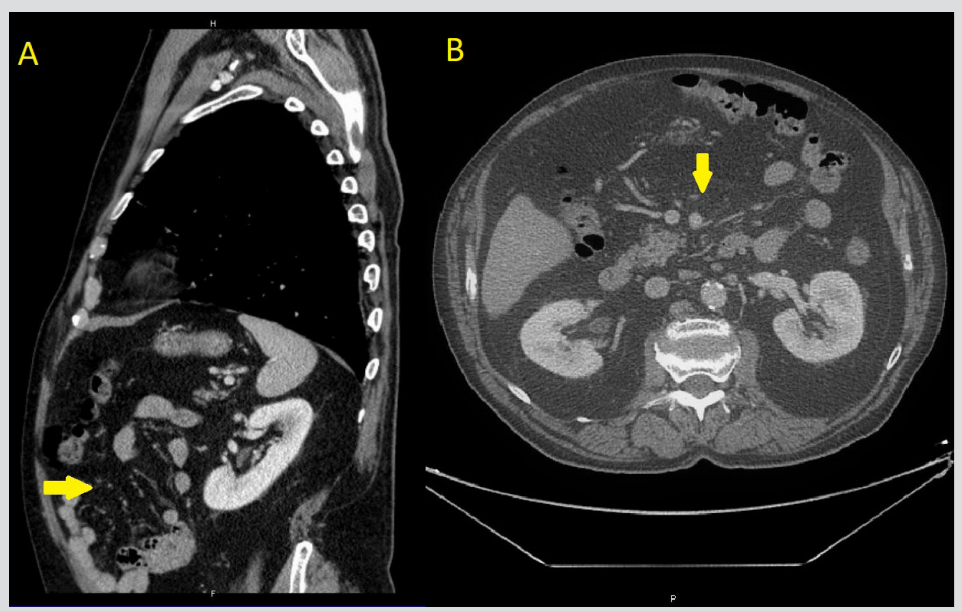

Figure 1. (A,B) Mesenteric panniculitis (arrows) seen on abdominal CT scans.

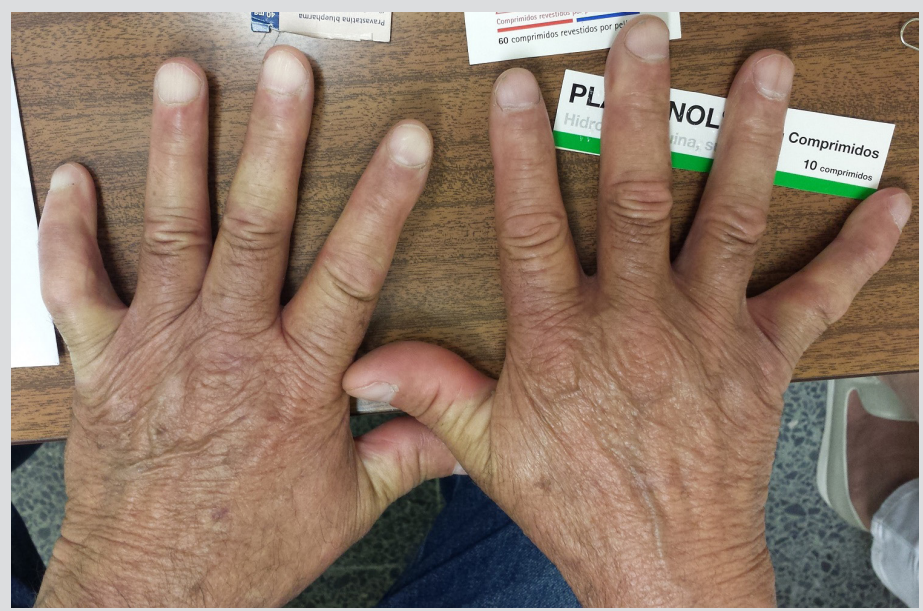

Figure 2. Raynaud's phenomenon with digital thickening

Blood tests showed the following abnormalities: mild thrombocytopenia, and mild elevation of alanine aminotransferase, aspartate aminotransferase and gamma glutamyl transferase. Serum autoimmunity tests showed anti-nuclear antibody (ANA) standard mottled titre of 1/1280 and nucleolar standard titre of 1/320, positive homogenous nucleoli, positive antibody anti-Sjögren's syndrome-related antigen A (anti-SSA) 60, strong positive SSA 52 and anti-Sjögren's syndrome related antigen B (anti-SSB), anti-Ku antibody weakly positive and antidouble stranded DNA (anti-ds-DNA) of $5.49 \mathrm{IU} / \mathrm{ml}$ (reference range $<5 \mathrm{IU} / \mathrm{ml}$ ). Serum protein electrophoresis, complements and 24-hour urinalysis were normal and viral serologies including hepatitis C virus antibodies (anti-HCV) were negative. 
Biopsy of minor salivary glands revealed lymphocytic sialoadenitis with normal parotid scintigraphy and oesophageal manometry. An echocardiogram showed dilation of the aortic root and left cavities, without signs of pulmonary hypertension. A thoraco-abdomino-pelvic CT scan revealed multiple small cystic formations juxtaposed in the pulmonary parenchyma, with variable size and thickened wall with subpleural predominance accompanied by traction bronchiolectasis, reaching all lobes, indicating usual interstitial pneumonia (UIP) (Fig. 3). Steatotic infiltration was seen in the liver and fat infiltration with small ganglion formations in the mesentery were observed, compatible with mesenteric panniculitis. Lung function tests showed mild restrictive syndrome with impaired diffusion. Finally, the capillaroscopy showed relatively well-preserved capillary distribution with no evident loss of capillaries; however, spontaneous capillary haemorrhages (Fig. 4A), slow flow, irregular enlargement of the capillaries with absent giant capillaries (Fig. 4B) and significant oedema were seen.

The diagnosis of primary SS was made according to the 2016 ACR/EULAR (American College of Rheumatology/European League Against Rheumatism) criteria. The case was discussed in a team meeting, and it was decided not to initiate corticosteroid therapy since the patient did not have abdominal pain. He started hydroxychloroquine ( $200 \mathrm{mg} / \mathrm{day}$ ) and calcium channel blockers for Raynaud's phenomenon.
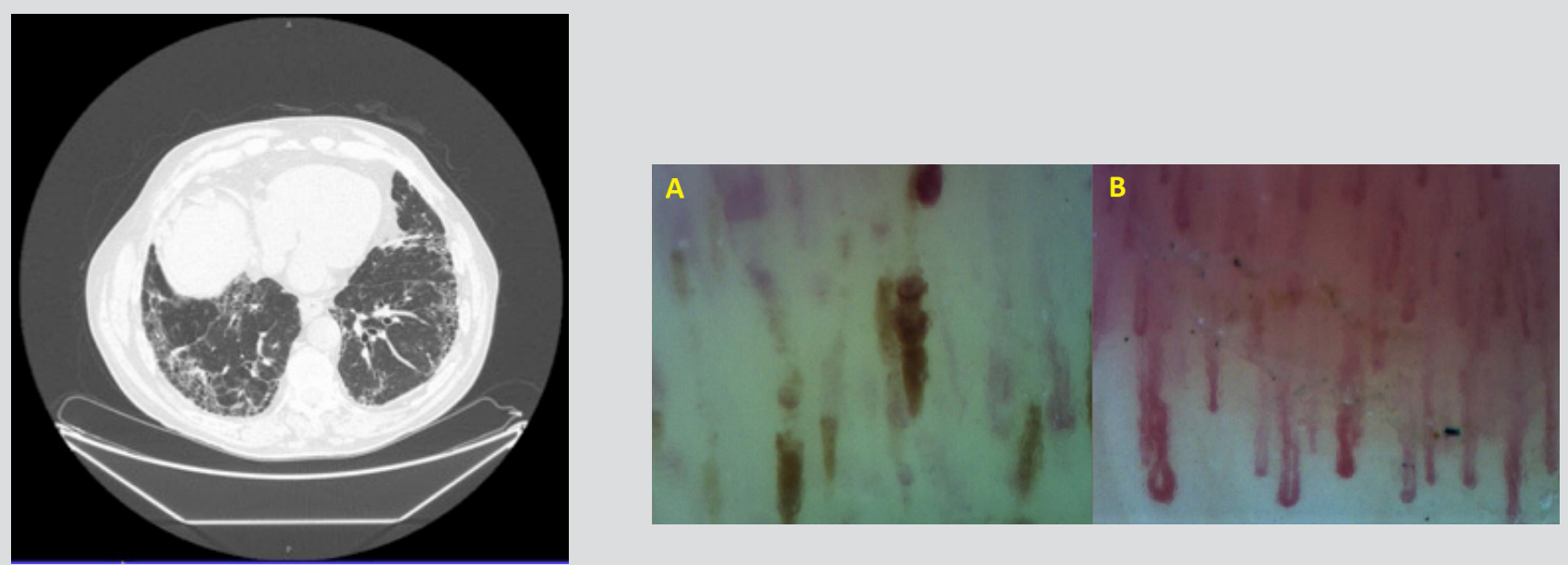

Figure 3. Multiple small cystic formations (arrows) juxtaposed in the pulmonary parenchyma, of variable size and with a thickened wall with Figure 4. (A) Spontaneous haemorrhages; (B) slow flow, dilated capillaries and oedema subpleural predominance accompanied by traction bronchiolectasis. The formations were present in all standard lobes and indicated usual interstitial pneumonia

\section{DISCUSSION}

Many conditions are linked to mesenteric panniculitis including autoimmune diseases ${ }^{[1]}$. However, SS is rarely associated with this condition, with less than 10 reported cases in the literature [3-6]. The majority of reported patients with mesenteric panniculitis needed treatment (corticosteroids and other immunosuppressants, or even surgery) as they were symptomatic. Clinical presentation ranges from general symptoms to gastrointestinal symptoms such as abdominal pain, nausea and vomiting or abdominal mass ${ }^{[1]}$. However, the discovery of $\mathrm{MP}$ in our patient was accidental, as he had no symptoms related to this condition, and so it was decided not to initiate immunosuppression. In this case, it was difficult to distinguish the aetiology of MP from SS or the patient's surgical history. The two latter entities together have been linked to this pathology previously ${ }^{[5]}$ and MP may be associated with SS in patients who have undergone surgery.

The question arises to what extent an asymptomatic condition should be investigated. Total costs with this patient were about $€ 770$ : $€ 274.50$ for autoimmunity investigations, $€ 100$ for blood tests, $€ 246.40$ for CT scans and an estimated $€ 150$ for the remaining examinations and cytological results. The patient had been referred to the clinic for a causal investigation but, after being told that he only needed to be kept under surveillance, both he and the family chose to continue the investigation. After a careful clinical history was taken at the first visit, the patient described several complaints, as mentioned above, which had not been referred to his general practitioner but which revealed symptomatic atypical SS with systemic involvement, presenting with UIP, restrictive syndrome and diffusion impairment, Raynaud's phenomenon and the asymptomatic mesenteric panniculitis. 


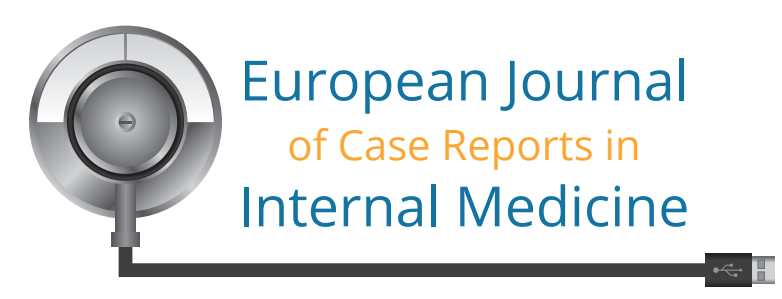

A patient sent for investigation of an asymptomatic pathology incurred a relatively high cost. In retrospect, serum protein electrophoresis, complements, 24-hour and viral serologies were not needed. However, most of the examinations were requested mainly for clarification of the other complaints which had not been previously referred. Nevertheless, considering the increasingly smaller health budgets, it is very important not to carry out unnecessary investigations, which is sometimes problematic owing to ill-defined clinical situations, increasingly informed patients, lack of time or clinical inexperience.

\section{REFERENCES}

1. Hussein MRA, Abdelwahed SR. Mesenteric panniculitis: an update. Expert Rev Gastroenterol Hepatol 2015;9:67-78.

2. Both T, Dalm VASH, Martin van Hagen P, Van Daele PLA. Reviewing primary Sjögren's syndrome: beyond the dryness - from pathophysiology to diagnosis and treatment. Int J Med Sci 2017;14:191-200.

3. Batten RL, Ng WF. A case report of mesenteric panniculitis and primary Sjögren's syndrome. OJRA 2013;3:ID 39729

4. Rios-Fernández R, Callejas-Rubio JL, Caba-Molina M, Ríos-Peregrina R, Ortego-Centeno N. Mesenteric inflammatory venoocclusive disease in a patient with Sjögren's syndrome. Case Rep Med 2014:ID 423420.

5. Burns RL, Bhavnagri SJ. Undiagnosed Sjögren's syndrome presenting as mesenteric panniculitis. Case Rep Rheumatol 2016 : ID 7207638.

6. Kakimoto K, Inoue T, Toshina K, et al. Multiple mesenteric panniculitis as a complication of Sjögren's syndrome leading to ileus. Intern Med 2016;55:131-134. 